COMMENT

Comment on 'On the dipole, velocity and acceleration forms in high-order harmonic generation from a single atom or molecule'

To cite this article: J A Pérez-Hernández and L Plaja 2011 J. Phys. B: At. Mol. Opt. Phys. 45028001

View the article online for updates and enhancements.
Related content

$$
\begin{aligned}
& \text { - On the dipole, velocity and acceleration } \\
& \text { forms in high-order harmonic generation } \\
& \frac{\text { from a single atom or molecule }}{\text { Jan Conrad Baggesen and Lars Bojer }} \\
& \text { Madsen } \\
& \text { - The } 1940 \text { Summer Meeting of the } \\
& \text { Astronomical Society of the Pacific (with } \\
& \text { Abstracts of the Papers) } \\
& \text { Gerald E. Kron } \\
& \text { - High-order harmonic generation from } \\
& \text { Confined Rydberg atoms } \\
& \text { Kudiyar Orazymbetov, Erdi A Bleda, Zikri }
\end{aligned}
$$

\section{Recent citations}

- Saddle point approaches in strong field physics and generation of attosecond pulses

Arjun Nayak et al 


\title{
Comment on 'On the dipole, velocity and acceleration forms in high-order harmonic generation from a single atom or molecule'
}

\author{
J A Pérez-Hernández ${ }^{1}$ and L Plaja ${ }^{2}$ \\ ${ }^{1}$ Centro de Láseres Pulsados CLPU, E-37008 Salamanca, Spain \\ 2 Grupo de Investigación en Óptica Extrema (GIOE), Universidad de Salamanca, Plaza de la Merced s/n, \\ E-37008 Salamanca, Spain \\ E-mail: joseap@usal.es
}

Received 28 July 2011

Published 22 December 2011

Online at stacks.iop.org/JPhysB/45/028001

\begin{abstract}
We show that the main conclusion of Baggesen and Madsen (2011 J. Phys. B: At. Mol. Opt.

Phys. 44 115601) is not general but only valid in one dimension.
\end{abstract}

\section{Introduction}

In [1], Baggesen and Madsen address the fundamental question on how to compute high-order harmonic generation from the time-dependent wavefunction $|\Psi(t)\rangle$, corresponding to the dynamics of an electron in an atom or molecule in the single active electron approximation. A main conclusion of this paper is that the harmonic spectrum is related directly to the dipole velocity. The argument is sustained on the integral solution of the one-dimensional wave equation (equation (5) in [1]); also the authors back up their results with a previous paper [3], which is believed to also relate the general problem of harmonic generation to the dipole velocity instead of the dipole acceleration. We claim that the conclusion in [1] is invalid in three dimensions, i.e. for any realistic computation of harmonic generation, and that the dipole spectrum is generally related with the dipole acceleration and not with the velocity. We also remark that the one-dimensional wave equation is not adequate for the case of an 'infinitely thin gas' referred in the abstract of the paper.

Classical electrodynamics gives an integral solution for the three-dimensional wave equation

$$
\nabla^{2} \mathbf{E}-\frac{1}{c^{2}} \frac{\partial^{2} \mathbf{E}}{\partial t^{2}}=\frac{1}{\epsilon_{0} c^{2}} \frac{\partial \mathbf{J}}{\partial t}
$$

of the form [2]

$$
\mathbf{E}(\mathbf{r}, t)=-\frac{1}{4 \pi \epsilon_{0} c^{2}} \int \frac{1}{\left|\mathbf{r}-\mathbf{r}^{\prime}\right|}\left[\frac{\partial}{\partial t^{\prime}} \mathbf{J}\left(\mathbf{r}^{\prime}, t^{\prime}\right)\right]_{t^{\prime}=t-\left|\mathbf{r}-\mathbf{r}^{\prime}\right| / c} \mathrm{~d} \mathbf{r}^{\prime},
$$

where it is already clear that the source of the field radiated by a moving charge is the time derivative of the current density, i.e. the charge acceleration. In our opinion, this solution being exact, there is little doubt that the harmonic spectrum radiated by a charge is related directly to the acceleration and not to the velocity, as it is concluded in [1]. The source of misunderstanding in [1] is the use of the one-dimensional wave equation (equation (5) in [1]) instead of the three dimensional, written here as equation (1).

The three-dimensional wave equation can be reduced to one dimension for the case of charge distributions uniform in the transversal plane $\mathbf{J}(x, y, z, t)=\mathbf{J}(x, t)$. Imposing this condition, equation (1) must lead to a particular solution with the same symmetry, i.e. $\mathbf{E}(x, t)$. Note that these charged planes are very different from the infinitely thin gas distribution' suggested in [1], which corresponds to $\mathbf{J}(x, y, z, t)=\lambda(x, t) \delta(y) \delta(z)$ ( $\lambda$ being a linear current density). In this latter case, the associated radiated field (2) remains three dimensional, i.e. not a solution of the onedimensional wave equation. 
The authors also point out that the association of radiation to the dipole velocity is also found in equation (7) in [3]. However, we should remark that this expression is specific to the generation of a plane wave, which is uniform transversally and therefore it can be reduced to a solution of the onedimensional wave equation.

To clarify our point, we will derive the solution (equation (6) in [1]) from the three-dimensional integral solution, equation (2), assuming a transversally uniform charge distribution $\mathbf{J}(x, t)$. Following [1] we will also assume $\mathbf{J}(x, t)=\dot{\mathbf{D}}(t) \delta(x)(\dot{\mathbf{D}}(t)$ being the dipole velocity per unit area. Substituting it in equation (2), we have

$$
\begin{aligned}
& \mathbf{E}(\mathbf{r}, t)=-\frac{1}{4 \pi \epsilon_{0} c^{2}} \iiint \frac{1}{\left|\mathbf{r}-\mathbf{r}^{\prime}\right|} \\
& \times\left[\frac{\partial}{\partial t^{\prime}} \dot{\mathbf{D}}(t) \delta\left(x^{\prime}\right)\right]_{t^{\prime}=t-\left|\mathbf{r}-\mathbf{r}^{\prime}\right| / c} \mathrm{~d} \varphi^{\prime} \rho^{\prime} \mathrm{d} \rho^{\prime} \mathrm{d} x^{\prime},
\end{aligned}
$$

where we use the cylindrical coordinates $\left(\rho^{\prime}, \varphi^{\prime}, x^{\prime}\right)$. Since the current density is transversally uniform, we are free to choose any coordinate origin. Therefore, we will choose it so that the detector is located at the $x$ axis: $\mathbf{r}=x \mathbf{e}_{x}$, then $\left|\mathbf{r}-\mathbf{r}^{\prime}\right|=\sqrt{\rho^{\prime 2}+\left(x^{\prime}-x\right)^{2}}$. Integrating (3) over the $x^{\prime}$ and $\varphi^{\prime}$ coordinates,

$$
\begin{aligned}
\mathbf{E}(\mathbf{r}, t) & =-\frac{1}{2 \epsilon_{0} c^{2}} \int \frac{1}{\sqrt{\rho^{\prime 2}+x^{2}}} \\
\times & {\left[\frac{\partial}{\partial t^{\prime}} \dot{\mathbf{D}}\left(t^{\prime}\right)\right]_{t^{\prime}=t-\sqrt{\rho^{\prime 2}+x^{2}} / c} \rho^{\prime} \mathrm{d} \rho^{\prime} . }
\end{aligned}
$$

From the definition of the retarded time, we have $\mathrm{d} t^{\prime}=$ $-\rho^{\prime} \mathrm{d} \rho^{\prime} / c \sqrt{\rho^{\prime 2}+x^{2}}$; therefore, the radial integral can be cast into an integral over $t^{\prime}$,

$$
\begin{aligned}
\mathbf{E}(x, t) & =-\frac{1}{2 \epsilon_{0} c} \int_{-\infty}^{t-|x| / c}\left[\frac{\partial}{\partial t^{\prime}} \dot{\mathbf{D}}\left(t^{\prime}\right)\right] \mathrm{d} t^{\prime} \\
& =-\frac{1}{2 \epsilon_{0} c} \dot{\mathbf{D}}\left(t-\frac{|x|}{c}\right),
\end{aligned}
$$

that corresponds to equation (6) in [1] ( $\dot{\mathbf{D}}$ is assumed 0 at $\left.t^{\prime} \rightarrow-\infty\right)$

To summarize, we find that (a) the harmonic spectrum is directly related to the dipole acceleration, as shown in equation (2), and not to the dipole velocity; (b) the conclusion in [1] assigning the dipole velocity as the source of harmonics is a consequence of considering the one-dimensional wave equation instead of the three dimensional one; (c) the solution found in [1] is only valid in three dimensions for the particular case of the radiation emitted by infinite charged transversal planes, with uniform transversal charge density; (d) the 'infinitely thin gas' suggested in [1] does not lead to a solution of the type of equation (6) in [1], since it cannot be reduced to a solution of the $1 \mathrm{D}$ wave equation.

\section{Acknowledgments}

Authors acknowledge support from Spanish Ministerio de Ciencia e Innovación through the Consolider Program SAUUL (CSD2007-00013) and research project FIS2009-09522, from Junta de Castilla y León through the Program for Groups of Excellence (GR27) and from the EC's Seventh Framework Programme (LASERLAB-EUROPE, grant agreement no 228334).

\section{References}

[1] Baggesen J C and Madsen L B 2011 On the dipole, velocity and acceleration forms in high-order harmonic generation from a single atom or molecule J. Phys. B: At. Mol. Opt. Phys. 44115601

[2] Jackson J D 1975 Classical Electrodynamics (New York: Wiley)

[3] Diestler D J 2008 Harmonic generation: quantum-electrodynamical theory of the harmonic photon-number spectrum Phys. Rev. A 78033814 